\title{
Role of subsurface physics in the assimilation of surface soil moisture observations *
}

\author{
Sujay V. Kumar a,b, Rolf H. Reichle ${ }^{\mathrm{c}}$, Randal D. Koster ${ }^{\mathrm{c}}$, \\ Wade T. Crow ${ }^{\mathrm{d}}$, Christa D. Peters-Lidard ${ }^{\mathrm{b}}$ \\ ${ }^{a}$ Science Applications International Corporation, Beltsville, $M D$ \\ ${ }^{\mathrm{b}}$ Hydrological Sciences Branch, NASA Goddard Space Flight Center, Greenbelt, \\ $M D$ \\ ${ }^{\mathrm{c}}$ Global Modeling and Assimilation Office, NASA Goddard Space Flight Center, \\ Greenbelt, $M D$ \\ ${ }^{\mathrm{d}}$ USDA-ARS Hydrology and Remote Sensing Laboratory, Beltsville, MD \\ Keywords
}

land surface modeling, data assimilation, remote sensing, hydrology, soil moisture, root zone 


\begin{abstract}
Root zone soil moisture controls the land-atmosphere exchange of water and energy and exhibits memory that may be useful for climate prediction at monthly scales. Assimilation of satellite-based surface soil moisture observations into a land surface model is an effective way to estimate large-scale root zone soil moisture. The propagation of surface information into deeper soil layers depends on the modelspecific representation of subsurface physics that is used in the assimilation system. In a suite of experiments we assimilate synthetic surface soil moisture observations into four different models (Catchment, Mosaic, Noah and CLM) using the Ensemble Kalman Filter. We demonstrate that identical twin experiments significantly overestimate the information that can be obtained from the assimilation of surface soil moisture observations. The second key result indicates that the potential of surface soil moisture assimilation to improve root zone information is higher when the surface to root zone coupling is stronger. Our experiments also suggest that (faced with unknown true subsurface physics) overestimating surface to root zone coupling in the assimilation system provides more robust skill improvements in the root zone compared with underestimating the coupling. When CLM is excluded from the analysis, the skill improvements from using models with different vertical coupling strengths are comparable for different subsurface truths. Finally, the skill improvements through assimilation were found to be sensitive to the regional climate and soil types.
\end{abstract}

‡ Corresponding author: S. V. Kumar. Tel. 001-301-286-8663.

Fax:001-301-286-8624, email: Sujay.V.Kumar@nasa.gov 


\section{Introduction}

Soil moisture plays an important role in controlling evaporation, plant transpiration, infiltration, and runoff, and consequently in modulating the partitioning of water and energy fluxes across the land-atmosphere interface. Moreover, root zone soil moisture provides a critical memory function in the climate system at monthly time scales. Characterization of soil moisture in the root zone is therefore important for many applications, including agricultural and water resources management, short and medium term meteorological and climate studies and flood/drought forecasting. (Koster et al. (2004); Oglesby (1991); Chen and Avissar (1994); Trier et al. (2004); Kumar et al. (2007))

Using observation-based surface meteorological data to drive land surface models in an uncoupled manner is a common approach used to generate spatially and temporally continuous estimates of land surface states, including soil moisture (Mitchell et al. (2004); Rodell et al. (2004); Kumar et al. (2006)). The estimates from these models, however, are uncertain because of errors in model parameters and forcing inputs and because of deficiencies in the model representation of land surface processes. Indirect estimates of surface soil moisture for the top 1-5 cm of the soil column are also available from satellite remote sensing observations (Schmugge et al. (1980); Engman and Gurney (1991); Jackson (1993); Njoku and Entekhabi (1995)). Such satellite retrievals, however, are subject to measurement noise and errors in retrieval 
models, are limited to the top few millimeters or centimeters of soil and do not provide complete spatial and temporal coverage. An effective way to attenuate model and observational errors and produce superior estimates of soil moisture states is to constrain the land model predictions with satellite observations of surface soil moisture through data assimilation methods. Such methods vertically extrapolate temporally intermittent surface retrievals and produce estimates of root zone soil moisture that are generally superior to estimates from land surface models alone (Reichle et al. (2007)).

Various computational techniques have been used to derive estimates of the soil moisture profile from surface measurements, including regression techniques, inversion of radiative transfer methods, parametric profile models and data assimilation methods in conjunction with physical models (Jackson (1986); Kostov and Jackson (1993); Jackson (1993); Entekhabi et al. (1994); Li and Islam (2002)). Among these efforts, the integrated use of data assimilation and hydrological models has been cited as the most promising approach. Some early feasibility and field-scale studies demonstrated improvements in near surface and bulk subsurface soil moisture through data assimilation (Calvet et al. (1998); Heathman et al. (2003); Montaldo et al. (2001); Reichle et al. (2002a); Walker et al. (2001, 2002); Reichle and Koster (2003)). Improvements in surface and root zone soil moisture through data assimilation of global satellite retrievals have recently been demonstrated (Reichle and Koster (2005); Reichle et al. (2007); Drusch (2007)). Taken together, these studies describe the development of 
advanced methodologies and establish the potential of near surface soil moisture data assimilation to infer estimates of subsurface profiles.

Data assimilation techniques rely on the inherent surface to root zone connection to propagate surface information to deeper soil layers. The subsurface physics used in the land surface model, therefore, is an important factor in determining the strength and validity of the downward propagation of surface information. In this article, we evaluate how the use of different subsurface physics impacts the data assimilation performance, especially in the root zone assimilation products. The experiment is conducted with four land surface models (LSMs) of varying complexity (Catchment, Mosaic, Noah, and CLM) - each applying different subsurface physics schemes. As we will show, the Catchment and Mosaic LSM exhibit particularly strong soil moisture coupling between its surface and root zones, while Noah, and CLM show successively weaker connections between the surface and root zone.

Synthetic observations generated from control integrations using each of the four models are re-assimilated back into the same model and cross-assimilated into each of the other three models. This setup leads to a suite of experiments where each LSM is provided with different sets of observations. Depending on the surface-root zone (vertical) coupling strength of the LSM, the information from surface observations is vertically propagated differently for each LSM during data assimilation. The evaluation of the assimilation products reveals how well each LSM performs in a data assimilation system under varying assumptions of vertical coupling strength. It must 
be stressed that the intent of the experiments is not to judge the veracity of the LSMs to reproduce large-scale land surface processes and conditions as they occur in nature. Again, our goal is to demonstrate how the LSMs perform in a data assimilation system under various different representations of possible true land surface processes. In particular, we aim to quantify how the strength of the vertical connection between the surface and root zone (in the assimilation model or in the assumed "truth") affects the efficiency and veracity of information transfer into the root zone through assimilation. Understanding this transfer is key to exploiting the information content of the next generation of satellite soil moisture retrievals from the Soil Moisture

Ocean Salinity (Space Studies Board (2007)) and the Soil Moisture Active-Passive (Kerr et al. (2001)) satellite missions to be launched in 2009 and 2013, respectively.

\section{Approach}

\subsection{Land Surface Models}

This study is conducted using the Land Information System (LIS) data assimilation testbed, which provides a framework for the integrated use of several community LSMs, observation types and sequential data assimilation algorithms (Kumar et al. (2008b)). The interoperable features of the LIS framework (Kumar et al. (2006); Peters-Lidard et al. (2007); Kumar et al. (2008a)) make it an ideal platform for 
conducting the intercomparison experiments presented here.

The suite of experiments presented in this article is conducted using four community LSMs: (1) NASA Catchment LSM (Koster et al. (2000)), (2) Mosaic LSM (Koster and Suarez (1996)), (3) Noah LSM (Ek et al. (2003)), and (4) Community Land Model (CLM) version 2.0 (Dai et al. (2003)). All four models dynamically predict land surface water and energy fluxes in response to surface meteorological forcing inputs, but differ in their structural representation of surface and subsurface water and energy balance processes.

Three of the four models are traditional land surface schemes that model soil moisture dynamics by solving a layer-based formulation of the standard diffusion and gravity drainage equations for unsaturated flow. Mosaic has three soil layers: a thin $2 \mathrm{~cm}$ surface layer, a $148 \mathrm{~cm}$ middle layer, and a $200 \mathrm{~cm}$ thick bottom layer. Noah uses 4 soil layers of increasing thicknesses of 10, 30, 60 and $100 \mathrm{~cm}$. CLM (as used here) employs a more highly discretized representation of the subsurface with 10 unevenly spaced layers. CLM's layers have thicknesses of 1.75, 2.76, 4.55, 7.5, 12.36, 20.38, 33.60, 55.39, 91.33 and $113.7 \mathrm{~cm}$, respectively.

The Catchment LSM, by contrast, is non-traditional in that the vertical soil moisture profile is determined through deviations from the equilibrium soil moisture profile between the surface and the water table. Soil moisture in a $2 \mathrm{~cm}$ surface layer and a $100 \mathrm{~cm}$ root zone layer is then diagnosed from the modeled soil moisture profile. 
The Catchment model includes an explicit treatment of the horizontal variation of soil water and water table depth within each hydrological catchment based on topographic variations within the catchment. Typically, the Catchment model is used with hydrologically defined catchments (or watersheds) as basic computational units. For ease of model intercomparison, however, the Catchment LSM is used on a regular latitude-longitude grid in this study.

For the remainder of the paper and clean comparison of output across LSMs, we define root zone soil moisture as the soil moisture content in the top $1 \mathrm{~m}$ of the soil column, regardless of the LSM and its (potentially different) native definition of the term. In other words, our root zone moisture content is derived as a suitably weighted vertical average over the model layers that are within the top $1 \mathrm{~m}$ of the soil column. By contrast, we refer to surface soil moisture as the top-most layer of each model. The specific layer depth for surface soil moisture is $2 \mathrm{~cm}$ for Catchment and Mosaic, $1.75 \mathrm{~cm}$ for CLM, and $10 \mathrm{~cm}$ for Noah.

\subsection{Ensemble Kalman Filter}

The Ensemble Kalman Filter (EnKF) is widely used as an effective technique for soil moisture assimilation (Reichle et al. (2002a,b); Crow and Wood (2003); Zhou et al. (2006)). The EnKF provides a flexible approach for incorporating errors in the model and the observations. Its ensemble-based treatment of errors makes it suitable for 
handling the modestly non-linear dynamics and the temporal discontinuities that are typical of land surface processes. We employ the EnKF approach in all the experiments presented in this article.

The EnKF alternates between an ensemble forecast step and a data assimilation update step (Reichle et al. (2002b)). In the forecast step, an ensemble of model states is propagated forward in time using the land surface model. In the update step at time $k$, this model forecast is adjusted towards the observation based on the relative uncertainties, with appropriate weights expressed in the "Kalman gain" $\mathbf{K}_{k}$ :

$$
\mathbf{x}_{k}^{i+}-\mathbf{x}_{k}^{i-}=\mathbf{K}_{k}\left[\mathbf{y}_{k}^{i}-\mathbf{H}_{k} \mathbf{x}_{k}^{i-}\right]
$$

The state and (suitably perturbed) observation vectors are represented by $\mathbf{x}_{k}$ and $\mathbf{y}_{k}$, respectively. The observation operator $\mathbf{H}_{k}$ relates the model states to the observed variable. The superscripts $i-$ and $i+$ refer to the state estimates of the $i$-th ensemble member $(-)$ before and $(+)$ after the update, respectively. Put differently, equation (1) states that the analysis increments $\left(\mathbf{x}_{k}^{i+}-\mathbf{x}_{k}^{i-}\right)$ are computed by multiplying the innovations $\left(\mathbf{y}_{k}^{i}-\mathbf{H}_{k} \mathbf{x}_{k}^{i-}\right)$ with the Kalman gain $\mathbf{K}_{k}$. The Kalman gain, in turn, is computed from the observation error covariance $\mathbf{R}_{k}$ and the forecast error covariance $\mathbf{P}_{k}^{-}$(diagnosed as the sample covariance of the ensemble of model forecasts):

$$
\mathbf{K}_{k}=\mathbf{P}_{k}^{-} \mathbf{H}_{k}^{T}\left[\mathbf{H}_{k} \mathbf{P}_{k}^{-} \mathbf{H}_{k}^{T}+\mathbf{R}_{k}\right]^{-1}
$$

Note that the key term $\mathbf{P}_{k}^{-} \mathbf{H}_{k}^{T}$ is the cross-covariance between errors in the model 
states (for example, surface and root zone soil moisture) and errors in the observed variable (that is, surface soil moisture), while the term in square brackets in equation (2) is essentially a normalization factor.

The successive model propagation and update steps imply that surface information is propagated into the root zone in two distinct ways. First, during the model propagation step, soil moisture is exchanged between the surface and deeper layers according to the modeled soil moisture dynamics, typically diffusion and gravity drainage. Second, whenever a surface soil moisture observation is available, an increment to deeper layer soil moisture is computed and applied in the EnKF update step, based on the surface innovation and the surface-root zone error correlation (as expressed in the Kalman gain). Given the time scales of soil moisture processes and the fact that the observed surface layer is typically thin compared to deeper soil column reservoirs, the propagation of surface information solely through vertical model physics is relatively inefficient. By contrast, the updating of deeper layer soil moisture based on the modeled surface-root zone error correlations (expressed in the ensemble) can provide for an efficient downward propagation of surface information, as long as errors in the surface layer are statistically connected to errors in deeper layers via the model physics. 


\section{Experiment Setup}

\subsection{Experiment Overview}

We designed a suite of synthetic experiments to investigate the influence of model representation of vertical water transport on assimilation performance. The basic structure of the experiments is as follows: A land surface model is selected and an ensemble integration (without data assimilation) is conducted. Each member of the ensemble experiences a different realization of synthetic errors in the forcing inputs and the model prognostic variables (see below). From this ensemble, a single realization (or ensemble member) is chosen and assumed to represent the "true" state of the land surface, referred to as the control (or truth) run. This synthetic truth serves two purposes: (i) a subset of the "truth" surface soil moistures, consistent with satellite retrieval availability, is isolated, corrupted with synthetic observation errors, and then used for assimilation into the available land surface models, and (ii) the soil moistures produced in this truth, or "control", simulation are used to evaluate the accuracy of subsequent model integrations that assimilate synthetic observations generated from this truth. The mean over all members of the ensemble integration represents the "open loop" simulation and represents the model skill without the benefit of data assimilation. These steps are repeated for each LSM and yield four different sets of truth data, synthetic observations, and open loop estimates, one for each land surface model. 
Next, a given set of synthetic observations is assimilated into each of the four land surface models with the EnKF (section 2.2), resulting in four sets of data assimilation products, one for each model. Under our original assumption that a single member of the open loop LSM simulation (corresponding to the chosen set of synthetic observations) serves as "truth", we can then compute the skill with which the four assimilation integrations approximate the truth data. For a given model, the skill found for the assimilation product minus that for the corresponding open loop product is our metric of interest (section 3.3); this difference is the skill improvement associated with data assimilation. The process is then repeated, taking a simulation from a different model as "truth" and assimilating the corresponding synthetic observations into each of the four LSMs. After each of the four models serves in turn as "truth", we end up with a 4x4 matrix of skill improvement associated with data assimilation. The columns of the matrix represent the different versions of "truth". Each row of the matrix corresponds to a specific model used for assimilation, showing how assimilation improves its product relative to its openloop product under different versions of "truth".

Note that for a given assumed "truth", one of the four model experiments is an "identical twin" experiment, meaning that the model providing the truth is the same as that used in the assimilation integration. The other three experiments are referred to as "fraternal twin" experiments because they use an LSM in the assimilation system that is different from that which was used to generate the synthetic truth data for 
these experiments. This distinction is important in interpreting the matrix of results.

\subsection{Experiment Details}

All model and assimilation integrations are conducted over a gridded domain that roughly covers the Continental United States (CONUS, from $30.5^{\circ} \mathrm{N}, 124.5^{\circ} \mathrm{W}$ to $\left.50.5^{\circ} \mathrm{N}, 75.5^{\circ} \mathrm{W}\right)$ at $1^{\circ}$ spatial resolution. The LSMs are driven with meteorological forcing data from the Global Data Assimilation System (GDAS; the global meteorological weather forecast model of the National Center for Environmental Prediction (Derber et al. (1991))). First, the models are spun up by cycling three times through the period from 1 January 2000 to 1 January 2007. This ensures that the internal model prognostic states have adequate time to reach an equilibrium consistent with the model climatology, meteorological forcing and parameters. All model and assimilation integrations are conducted over the same 7-year period. To avoid potential assimilation-related spin-up effects, only data for the 6-year period from 1 January 2001 to 1 January 2007 are used in the subsequent analysis.

Each open loop or assimilation experiment with a given model consists of 12 ensemble members (Kumar et al. (2008b)), and all data assimilation estimates are based on taking a mean of the ensemble. The ensemble members differ from each other in two ways: (i) noise is added to the meteorological forcing, and (ii) noise is added to the model prognostic fields. The parameters used for these perturbations are listed in 
Tables 1a-1e. Zero-mean, normally distributed additive perturbations are applied to the downward longwave radiation forcing, and log-normal multiplicative perturbations with a mean value of 1 are applied to the precipitation and downward shortwave fields (Table 1a). Time series correlations are imposed via a first-order regressive model $(\mathrm{AR}(1))$ with a time scale of 24 hours. No spatial correlations are applied since this study uses the one-dimensional version of the EnKF (Reichle and Koster (2003)). Cross correlations are imposed on the perturbations of radiation and precipitation fields using the values specified in Table 1a.

Model prognostic variables for each LSM are perturbed with additive noise, with additional vertical correlations imposed on the perturbations for the Noah, CLM, and Mosaic LSMs prognostic variables. The parameters for the Catchment LSM (Table 1b) are based on the values of Reichle et al. (2008). The parameters for the other land surface models (Tables 1c-1e) are designed to yield comparable ensemble spreads and comparable open loop skills. For all model prognostic perturbations we impose AR(1) time series correlations with a 12 hour time scale. Further, it was also ensured that these error settings do not introduce systematic biases in the truth and open loop integrations relative to the standard, unperturbed model integrations.

For the assimilation experiments, the synthetic soil moisture retrievals require some special preprocessing. To account for difficulties in retrieving soil moisture products from microwave sensors, the synthetic observations are masked out for high vegetation density (specifically, when the Green Vegetation Fraction values used in Noah exceed 
0.7). Also, the soil moisture "observations" are masked out when snow is present on the ground, when the soil is frozen and during precipitation events, to mimic the difficulty of retrieving soil moisture during these events. The data masks for snow and frozen soil are generated based on the snow cover and soil temperature values from the control integrations of all four models. Further, random Gaussian noise with an error standard deviation of $0.03 \mathrm{~m}^{3} \mathrm{~m}^{-3}$ (volumetric soil moisture) is added to the synthetic observations to mimic measurement uncertainty.

Data assimilation methods (including the EnKF) are designed to correct random errors in the model background and assume that model and observations are climatologically unbiased. The climatologies of the model and satellite estimates, however, are typically very different, as are the climatologies of estimates from different land surface models. Such climatological biases must be addressed as part of the assimilation experiment. Here, we adopt the a priori scaling method of Reichle and Koster (2004). In this approach, the observations are scaled to the model's climatology so that the cumulative distribution functions (cdfs) of the observations and the model match (for each grid point). The scaling of observations is done prior to each assimilation experiment (except for identical twin experiments, where this scaling is not necessary because the observations are generated from the same land surface model that is used in the assimilation integration). Cdf matching can be used with new satellite sensors only after robust cdf estimates have been obtained. Reichle and Koster (2005) show that data records of 1 year are adequate. 


\subsection{Evaluation Metric: Normalized Information Contribution}

Since the observations are scaled prior to the assimilation experiment, the anomaly time series correlation (rather than RMSE) is used quantify the skill of the estimates. This anomaly time series is obtained (for each grid point) as follows. First, we subtract the monthly mean climatology of each dataset from the corresponding daily average raw data, so that the anomalies represent the daily deviations from the mean seasonal cycle. We thus do not take advantage of the "skill" inherent in the seasonal cycle. Subsequently, we compute the time series correlation coefficient between the daily anomaly estimates and the corresponding anomalies of the truth data, at each grid point. Note that only grid points with a minimum of 600 valid observations for the evaluation period are included in the comparisons.

To evaluate improvements due to assimilation, a normalized information contribution (NIC) metric is computed as follows: First, the monthly anomaly time series coefficients $R_{a}$ for the assimilation and $R_{o}$ for the open loop integrations are computed. A normalized information contribution is then defined as NIC $=\left(R_{a}-R_{o}\right) /\left(1-R_{o}\right)$, which is a measure of how much of the maximum skill improvement $\left(1-R_{0}\right)$ is realized through data assimilation $\left(\mathrm{R}_{\mathrm{a}}-\mathrm{R}_{\mathrm{o}}\right)$. Assuming that the assimilation product is no worse than the model-only output $\left(R_{\mathrm{a}}>\mathrm{R}_{\mathrm{o}}\right)$, we have $0 \leq \mathrm{NIC} \leq 1$. For $\mathrm{NIC}=0$, the assimilation of surface soil moisture does not add any information to the assimilation product, and for $\mathrm{NIC}=1$, the assimilation realizes the maximum skill 
improvement possible. The NIC metric is needed primarily because it is extremely difficult, if not impossible, to achieve identical open loop skill for different LSMs for all the 16 assimilation simulations. Hereinafter, we also refer to the NIC metric loosely as the "skill improvement" through data assimilation.

\section{Results and Discussion}

Before analyzing the contribution of the surface retrievals in the data assimilation system, it is informative to take a closer look at how surface soil moisture is connected to root zone soil moisture in the four LSMs, and how errors in the surface layer are connected to errors in the root zone.

\subsection{Vertical Coupling Strength and Gain Correlation}

As discussed earlier, each land surface model possesses a different representation of soil moisture dynamics, based on its particular parameterizations of soils and vegetation properties, and processes related to the partitioning of rainfall into infiltration, runoff, and evaporation components. As a result, the coupling between the surface and subsurface soil moisture is different in each LSM. One way of measuring the vertical coupling strength is through correlating soil moisture anomalies in the surface layer with anomalies in root zone soil moisture. More precisely, we define the (spatially distributed) "native vertical coupling strength" as the anomaly correlation 
coefficient between surface and root zone soil moisture time series, for a given truth model integration without data assimilation. Put differently, the native vertical coupling strength measures the degree to which a positive (negative) anomaly in surface soil moisture coincides with a positive (negative) anomaly in the root zone.

This native vertical coupling strength is shown in Figure 1 for each of the four LSMs, by only including the locations and times at which surface soil moisture retrievals are available. Our subsequent analysis of the data assimilation performance follows a similar strategy, meant to characterize the skill improvements only at observation times and locations. Figure 1 shows that the surface and root zone soil moisture are most tightly coupled in the Catchment model, followed by Mosaic and Noah. CLM has the weakest coupling strength, possibly due to its use of the most soil layers. In other words, for soil moisture produced by the Catchment model, knowledge of a surface anomaly is more informative about root zone anomalies (at a given point in time) than for the other LSMs. Across all models, the native vertical coupling strength tends to be somewhat larger in the south and in the east of the domain, which is likely influenced by the generally wetter climate and the relative absence of cold-season processes. Note again that we only compare the coupling strength between different models and do not claim that a particular model has the least or most realistic representation of the coupling strength that occurs in nature.

The native coupling strength is an important metric that diagnoses the connection between surface and root zone soil moisture. It does not, however, directly measure 
how much a surface observation contributes to an update of root zone soil moisture in the EnKF. The surface-root zone connection in the data assimilation update is based on the modeled error correlations and can be diagnosed by a closer look at the Kalman gain. In the assimilation update step, the EnKF method computes analysis increments for surface and root zone soil moisture based on the Kalman gain and the innovations (equations (1) and (2)). Because we use a "one-dimensional" EnKF (Reichle and Koster (2003)), the observations are effectively scalars and the gain is a vector. The element $K_{j}$ of the gain that corresponds to a particular (model-specific) soil moisture layer $j$ is thus directly proportional to the error covariance between the model forecast soil moisture in the surface layer and that in layer $j$, labeled $x_{s f}$ and $x_{j}$, respectively:

$$
K_{j} \propto \operatorname{cov}\left(x_{s f}^{-}, x_{j}^{-}\right)
$$

The $K_{j}$ 's can easily be calculated from the ensemble at each update time during each assimilation integration. Next, we compute a (spatially distributed) scalar root zone gain $K_{r z}$ for the top $100 \mathrm{~cm}$ root zone layer through model-specific vertical averaging of the $K_{j}$ 's. Hereinafter, $K_{r z}$ is referred to as the "gain correlation" metric. It is determined primarily by the model physics and by our choice of perturbation input parameters (in particular the vertical correlations in the perturbations to the soil moisture states listed in Tables 1b to 1e). Most importantly, the gain correlation directly indicates the size of the root zone increment that results from a unit innovation 
and measures by how much a surface observation impacts adjustments of root zone soil moisture through the EnKF update.

Figure 1 also shows the time-average gain correlation for each LSM (averaged over the four assimilation experiments for which the given LSM was used in the assimilation system). The gain correlation trends are similar to the trends observed in the native vertical coupling strengths of each LSM, with Catchment having the highest value and Mosaic, Noah and CLM having successively lower values. Across all models, the gain correlations exhibit slightly larger values in the southern and eastern parts of the domain. The comparison of the vertical coupling strength and gain correlations in Figure 1 suggests that in assimilation integrations using a LSM with strong surfaceroot zone coupling (Catchment or Mosaic for example), root zone increments tend to correlate strongly with surface innovations. Similarly, using a LSM with weaker surface to root zone coupling is likely to produce less correlation between root zone increments and surface innovations.

\subsection{Assimilation Performance}

Let us now turn to the analysis of skill improvement through assimilation of surface observations. Tables 2 and 3 list the skill improvement (as domain averaged NIC values, section 3.3) for the surface and root zone soil moisture products, respectively. As mentioned earlier, the NIC values are computed using anomalies at times and 
locations for which surface soil moisture retrievals are available, representing skill improvements relative to possible observation instances.

Each table presents the domain averaged NIC values obtained from all 16 assimilation experiments, constituting the $4 \times 4$ matrix of skill improvements described in section 3. Again, the diagonal elements of this matrix represent the identical twin experiments and the off-diagonal elements represent the fraternal twin experiments. Note first that the skill improvements from the identical twin experiments are generally larger than those from the fraternal twin experiments, for both surface and root zone products. On average, the NIC values on the diagonal (corresponding to identical twin experiments) exceed the off-diagonal elements of the corresponding fraternal twin experiments by 0.18 for surface soil moisture improvements and by 0.24 for root zone soil moisture improvements. In a relative sense, identical twin experiments overestimate the skill derived from the assimilation of surface observations by 42 percent for surface soil moisture estimates and by 71 percent for root zone soil moisture estimates. Our first important conclusion is therefore that the identical twin experiments significantly overestimate the benefits derived from data assimilation relative to the fraternal experiments, which are more likely to represent the assimilation of actual satellite observations.

Each column of Tables 2 and 3 represents the benefit of surface soil moisture assimilation under a given scenario of true soil moisture physics, as obtained from different model representations. Correspondingly, the rows of Tables 2 and 3 measure the im- 
provements from assimilation of surface observations into a particular LSM for a range of potential "truths". If one assumes that each synthetic truth is equally likely, the mean over the row values represents an "expected value of skill improvement" in a data assimilation system that uses a particular LSM as its land model component. We have no way, of course, of justifying the assumption of equal likelihood here. We can say, though, that the spreads in the averages are larger for Table 3 than for Table 2, suggesting that while the ability of the LSMs to generate surface soil moisture information is comparable, model skill with regard to capturing root zone information varies significantly.

In conjunction with Figure 1, the Tables 2 and 3 suggest that the skill improvements in the root zone for a given location can be represented as a function of two factors: (1) the vertical coupling strength of the model used to generate the truth (hereafter referred to as VCS-truth) and (2) the vertical coupling strength of the model used in the assimilation system (hereafter referred to as the VCS-assimilation). To investigate this point further, Figure 2 stratifies ("bins") the root zone NIC values for all spatial locations and for all 12 fraternal twin assimilation integrations based on these two factors. (We exclude the results from the identical twin experiments, since they overestimate the skill improvements from data assimilation. If we had included them, only the diagonal points in the figure would change; the values along the diagonal would indeed increase, but the overall trends seen would remain the same.) The diagonal (shown in Figure 2) from the lower left corner to the upper right corner represents 
the skill improvement values when the VCS-model and VCS-assimilation values are roughly the same. On or off the diagonal, the skill improvements are generally higher for higher values of VCS-truth and VCS-assimilation, as indicated by the upper right corner in Figure 2.

This result is intuitive. Recall from Figure 1 that Catchment and Mosaic LSMs exhibit higher positive gain correlations compared to Noah and CLM LSMs, which implies that positive surface soil moisture innovations in Catchment and Mosaic lead to correspondingly positive increments in the root zone. Now recall that there is a strong correlation between the surface and root zone soil moisture in the Catchment and Mosaic LSM (as measured by the model's native coupling strength, Figure 1). When the LSMs with strong VCS serves as the truth, the assimilation system tends to produce root zone increments with the appropriate sign. This implies that the assimilation system does not need to rely as much on the less efficient process of propagating the surface increments into the root zone through the model physics. In other words, stronger vertical coupling makes it easier for the assimilation system to infer the root-zone estimates from the surface information. This trend is also consistent with Table 3, where the column averages of NICs are higher for Catchment and Mosaic truths, which have stronger vertical coupling strengths compared to Noah and CLM truths.

Simply put, if "truth" and the model in the assimilation system both show a strong connection between the surface and root zone (i.e, a strong VCS), surface information 
is more efficiently transferred to the root zone, increasing the skill scores. Figure 2 serves to quantify this intuitive result with an ensemble of models and data assimilation techniques.

The trends in Figure 2 also indicate a slight asymmetry in the NIC surface with the upper triangular area (relative to the lower left-upper right diagonal) showing higher NIC values compared to the lower triangular area. This implies that, for a given VCS-truth, the use of a model with higher native vertical coupling strength in the assimilation system tends to produce stronger skill improvements. This suggests that unless it is clear that a weak surface to root zone representation is the best modeling strategy, it is prudent to use a LSM with strongly coupled surface and root zone in the data assimilation. It must be noted that this inference is a direct result of the inclusion of CLM in the analysis. As evident from Table 3, the NIC values tend to be lower in the fraternal twin experiments with CLM as the assimilation model. We speculate that the highly discretized soil profile representation of CLM contributes to its relatively lower VCS. This hypothesis can be tested by changing the layering structure of a LSM and is left for a future research study. When CLM is excluded from the above analysis, the asymmetry is no longer observed in Figure 2 (Again, our analysis does not suggest that CLM represents natural processes particularly well or particularly poorly.) Another interesting trend to note is that the even when the assimilation model overestimates the truth vertical coupling strength by up to 0.1 , the skill improvements from assimilation still shows an increase as the VCS-truth 
increases.

To compute the statistical significance of the NIC values, the $99 \%$ confidence intervals of the anomaly time series correlation coefficients for the assimilation $\left(\delta \mathrm{R}_{\mathrm{a}}\right)$ and the open loop integrations $\left(\delta \mathrm{R}_{\mathrm{o}}\right)$ are translated into a corresponding $99 \%$ confidence interval for the NIC values $(\delta \mathrm{NIC})$ using equation 4.

$$
\delta \mathrm{NIC}=\delta \mathrm{R}_{\mathrm{a}} \frac{1}{\left(1-\mathrm{R}_{\mathrm{o}}\right)}+\delta \mathrm{R}_{\mathrm{o}} \frac{\left(\mathrm{R}_{\mathrm{a}}-1\right)}{\left(1-\mathrm{R}_{\mathrm{o}}\right)^{2}}
$$

Using this formulation, the $99 \%$ confidence intervals computed for the NIC values provide a range of approximately \pm 0.002 , indicating a high level of statistical significance in the skill improvement trends presented in Figure 2 and tables 2 and 3.

The dependence of the skill improvements from the assimilation runs to different climate regions is examined by stratifying the domain geographically. Figure 3 shows the average NIC values from different LSMs (averaged over the rows of the $4 \mathrm{x} 4$ matrix as in the "unknown truth" scenario) for five different geographic regions. (Note that the region in the North East location is omitted since there are not enough valid observation retrievals in this area). For each LSM, the trends in the skill improvements are similar across the five regions. In Catchment and Mosaic LSM, the magnitude of skill improvements in the root zone is comparable to the improvements in surface soil moisture, whereas for Noah and CLM, the root zone skill improvements are smaller than the surface skill improvements. This trend is consistent with our earlier result 
that models with strong vertical coupling are likely to generate root zone skill improvements more strongly correlated with surface skill improvements. In the three southern regions, the skill improvements generally increase going from west to east, consistent with the generally drier climate in the west compared to the generally wetter climate in the east. The wetter conditions lead to more tightly coupled surface and root zone conditions, which are easier to replicate as evident in Figure 2. Further, the skill improvements in the Northern regions are marginally lower than the corresponding values in the Southern regions. This could be due to the additional interaction of cold season processes and soil moisture dynamics that may lead to a decoupling of the surface and root zone soil moisture for part of the year.

The strength of coupling between different soil layers is also influenced by the soil texture types used in the models (Capehart and Carlson (1997)). Figure 4 shows a comparison of NIC values stratified according to the soil texture types in the domain. The skill improvements corresponding to sandier soils (loamy sand, sandy loam, sandy clay loam) are smaller compared to the improvements in clayey soils (loam, clay loam, clay). The clayey soils exert stronger capillary forces than sandy soils, and therefore show more tightly correlated surface and root zone improvements in all LSMs. 


\section{Summary}

Here we investigate the impact of various land surface model physics on soil moisture products derived through the assimilation of surface soil moisture retrievals. In the assimilation system, observed surface information is propagated into deeper soil layers, giving the surface retrievals an otherwise unobtainable relevance to such applications as the initialization of weather and seasonal climate forecasts. Since the LSMs differ significantly in their representation of subsurface water dynamics, the downward propagation of the surface information in the assimilation system strongly depends on which LSM is used as the system's model component. Here we study how the specific formulation of the LSM that is used in the assimilation system impacts the information contribution to soil moisture assimilation products.

The experiments presented in this article were conducted with the Catchment, Mosaic, Noah and CLM land surface models and the EnKF data assimilation algorithm. The modeling domain roughly covers the continental United States for a 6-year period. The LSMs vary in complexity in their representation of subsurface soil moisture dynamics. The Catchment LSM essentially describes deviations from the equilibrium soil moisture profile and has a relatively strong vertical coupling between the surface and root zone soil moisture. By contrast, the layer-based models Mosaic (3 layers), Noah (4 layers) and CLM (10 layers) have successively weaker coupling between their surface and root zones. 
Our synthetic experiments consisted of assimilating each of four synthetic retrievals data sets (based on integrations of each of the four LSMs) into four separate EnKFbased assimilation systems that use the four LSMs as their model component. The resulting 16 assimilation soil moisture products were evaluated against the corresponding synthetic truth data sets and compared to corresponding model integrations without the benefit of data assimilation. This information was summarized in a skill improvement metric that measures the normalized information contribution of the surface soil moisture retrievals to the skill of the soil moisture assimilation products (relative to the maximum possible improvement). This experiment setup allowed us to investigate the information contribution under a variety of combinations of possible true soil moisture dynamics with assimilation systems that use a range of LSMs.

The results clearly demonstrate that the assimilation of surface soil moisture provides improvements in the root zone estimates. The magnitude of the improvements depends on the LSM that is used in the assimilation system and on the (synthetic) true subsurface physics (that is, on the LSM that is used to generate the synthetic truth and the corresponding synthetic retrievals). Generally, identical twin experiments tend to overestimate skill improvements when compared to those of more realistic fraternal twin experiments. Likewise, the potential for improvements in the root zone is generally higher if the true subsurface physics exhibits a strong correlation between the surface and root zone, especially if the assimilation model also shows such a strong correlation. For weaker surface to root zone coupling strength, 
surface soil moisture assimilation yields more limited improvements in the root zone.

The results also provide insights into the optimal choice of LSM for soil moisture assimilation when the true subsurface physics is essentially unknown. An LSM with a strongly coupled representation of the surface and subsurface is perhaps a more robust choice for assimilation, unless independent information suggests that the use of a LSM with a more decoupled surface-subsurface representation is more realistic. We must emphasize here, however, that appropriate independent information (e.g. from soil moisture observations) is essentially unavailable. Point measurements of soil moisture exist but are not necessarily representative of large-scale vertical coupling strength. At large scales, the connection between the surface and root zone must be controlled in part (and probably enhanced) by lateral flow induced by topography and must, in any case, be affected by spatial heterogeneity in surface properties. Arguably, the "true" vertical coupling strength in nature for large-scale areas is unknown at this time.

The improvements in the soil moisture products through assimilation were found to be sensitive to the local climate and also the soil types used in the land surface models, which can in turn be explained by the dependence of the models' vertical coupling strength on soil type and regional climate. A statistical analysis of the computations demonstrates a high degree of statistical significance in the skill improvement values, and correspondingly in the trends demonstrated in the article. 
The comparison of the performance of different land surface models in response to the assimilation of surface soil moisture observations presented in this study is enabled by the LIS framework, which provides a unique environment for such a uniform intercomparison. The capabilities in LIS to use different forcing datasets, observations, and land surface models in an interoperable manner has enabled the rapid specification, calibration and application of the land surface models for data assimilation. The methodology demonstrated here with the LIS framework can be used as a guideline to evaluate the feasibility of using a land surface model for soil moisture assimilation. The procedure also provides a way to generate realistic measures of skill improvements from soil moisture assimilation, different from the identical twin experiment setup typically used to calibrate the assimilation system. Finally, the insights obtained on each models' performance through this study is expected to aid in their application for real assimilation experiments.

\section{Acknowledgements}

This research was partly supported by an internal investment grant from the NASA Goddard Space Flight Center. We also acknowledge the support from the Air Force Weather Agency that funded the development of data assimilation capabilities in LIS. Rolf Reichle was partly supported through NASA grants NNG05GB61G and NNX08AH36G. Computing was supported by the NASA High End Computing Pro- 
gram. We gratefully acknowledge this support.

\section{References}

Calvet, J.-C., J. Noilhan, and P. Bessemoulin. "Retrieving root-zone soil moisture from surface soil moisture of temperature estimates: a feasibility study based on field measurements." Journal of Applied Meteorology 37, 4: (1998) 371-386.

Capehart, W.J., and T.N. Carlson. "Decoupling of Surface and Near-Surface Soil Water Content: A Remote Sensing Perspective." Water Resources Research 33, 6: (1997) 1383-1395.

Chen, F., and R. Avissar. "The impact of land-surface wetness heterogeneity on mesoscale heat fluxes." Journal of Applied Meteorology 33, 11: (1994) 1323-1340.

Crow, W.T., and E.F. Wood. "The assimilation of remotely sensed soil brightness temperature imagery into a land surface model using ensemble kalman filtering: A case study based on ESTAR measurements during SGP97." Advances in Water Resources 26: (2003) 137-149.

Dai, Y., X. Zeng, R.E. Dickinson, I. Baker, G. Bonan, M. Bosilovich, S. Denning, P. Dirmeyer, P. Houser, G. Niu, K. Oleson, A. Schlosser, and Z.-L. Yang. "The common land model (CLM)." Bulletin of the American Meteorological Society 84, 4: (2003) 1013-1023. DOI:10.1175/BAMS-84-8-1013.

De Lannoy, G., R.H. Reichle, P.R. Houser, V. Pauwels, and N. Verhoest. "Correcting for forecast bias in soil moisture assimilation with the ensemble kalman filter." 
Water Resources Research 43, W09410.

Derber, J.C., D.F. Parrish, and S.J. Lord. "The new global operational analysis system at the National Meteorological Center." Weather and Forecasting 6: (1991) $538-547$.

Drusch, M. "Initializing numerical weather prediction models with satellite-derived surface soil moisture: Data assimilation experiments with ECMWF's Integrated Forecast System and the TMI soil moisture dataset." Journal of Geophysical Research 112, D03102.

Ek, M.B., K.E. Mitchell, L. Yin, P. Rogers, P. Grunmann, V. Koren, G. Gayno, and J.D. Tarpley. "Implementation of Noah land-surface model advances in the NCEP operational mesoscale Eta model." Journal of Geophysical Research 108, D22: (2003) doi:10.1029/2002JD003,296.

Engman, E. T., and R. J. Gurney. Remote Sensing in hydrology. London: Chapman and Hall, 1991.

Entekhabi, D., H. Nakamura, and E. G. Njoku. "Solving the inverse problem for soil moisture and temperature profiles by sequential assimilation of multifrequency remotely senses observations." IEEE Transactions on Geoscience and Remote Sensing 32: (1994) 438-448.

Heathman, G. C., P. J. Starks, L. R. Ahuja, and T. J. Jackson. "Assimilation of surface soil moisture to estimate profile soil water content." Journal of Hydrology 279: (2003) 1-17. 
Jackson, T. J. "Measuring surface soil moisture using passive microwave remote sensing." Hydrological Processes 7: (1993) 139-152.

Jackson, T.J. "Soil water modeling and remote sensing." IEEE Transactions on Geoscience and Remote Sensing GE-24, 1: (1986) 37-46.

Kerr, Y. H., P. Waldteufel, J.-P. Wigneron, J.-M. Martinnuzzi, J. Font, and M. Berger. "Soil moisture retrieval from space: The soil moisture and ocean salinity (SMOS) mission." IEEE Transactions on Geoscience and Remote Sensing 39, 8: (2001) $1729-1735$.

Koster, R., M. Suarez, P. Liu, U. Jambor, A. A. Berg, M. Kistler, R. H. Reichle, M. R. Rodell, and J. Famiglietti. "Realistic initialization of land surface states: Impacts on subseasonal forecast skill." Journal of Hydrometeorology 5: (2004) 1049-1063. Doi:10.1175/JHM-387.1.

Koster, R. D., M. J. Suarez, A. Ducharne, M. Stieglitz, and P. Kumar. "A catchmentbased approach to modeling land surface processes in a general circulation model 1. Model Structure." Journal of Geophysical Research 105, D20: (2000) 24,80924,822 .

Koster, R.D., and M.J. Suarez. "Energy and Water Balance Calculations in the Mosaic LSM." Technical memorandum 104606, NASA Goddard Space Flight Center, 1996.

Kostov, K. G., and T. J. Jackson. "Estimating profile soil moisture from surface layer measurements - a review." SPIE 1941: (1993) 125-136. 
Kumar, S.V., C.D. Peters-Lidard, J. L. Eastman, and W.-K. Tao. "An integrated high resolution hydrometeorological modeling testbed using LIS and WRF." Environmental Modelling and Software 23, 2: (2007) 169-181.

Kumar, S.V., C.D. Peters-Lidard, T. Tian, P.R. Houser, J. Geiger, S. Olden, L. Lighty, J.L. Eastman, B. Doty, P. Dirmeyer, J. Adams, K. Mitchell, E.F. Wood, and J. Sheffield. "Land information system: An interoperable framework for high resolution land surface modeling." Environmental Modeling and Software 21: (2006) $1402-1415$.

Kumar, S.V., C.D. Peters-Lidard, Y. Tian, R. H. Reichle, C. Alonge, J. Geiger, J. Eylander, and P. Houser. "An integrated hydrologic modeling and data assimilation framework enabled by the Land Information System (LIS)." IEEE Computer 41: (2008a) 52-59.

Kumar, S.V., R.H. Reichle, C.D. Peters-Lidard, R.D. Koster, X. Zhan, W.T. Crow, J.B. Eylander, and P.R. Houser. "A land surface data assimilation framework using the Land Information System: Description and Applications." Advances in Water Resources 31: (2008b) 1419-1432. Doi:10.1016/j.advwatres.2008.01.013.

Li, J., and S. Islam. "Estimation of root zone soil moisture and surface fluxes partitioning using near surface soil moisture measurements." Journal of Hydrology 259: (2002) 1-14.

Mitchell, K. E., D. Lohmann, P. R. Houser, J. C. Wood, E. F. Schaake, A. Robock, B. Cosgrove, J. Sheffield, Q. Duan, L. Luo, W. R. Higgins, R. T. Pinker, J. D. 
Tarpley, D. P. Lettenmaier, C. H. Marshall, J. K. Entin, M. Pan, W. Shi, V. Koren, J. Meng, B. H. Ramsay, and A. A. Bailey. "The multi-institution North American Land Data Assimilation System (NLDAS): utilization of multiple GCIP products and partners in a continental distributed hydrological modeling system." Journal of Geophysical Research 109: (2004) DOI:10.1029/2003JD003,823.

Montaldo, N., J. D. Albertson, M. Mancini, and G. Kiely. "Robust simulation of root zone soil moisture with assimilation of surface soil moisture data." Water Resources Research 37, 12: (2001) 2889-2900.

Njoku, E. G., and D. Entekhabi. "Passive microwave remote sensing of soil moisture." Journal of Hydrology 184: (1995) 101-130.

Oglesby, R. J. "Springtime soil moisture, natural climate variability, and North American drought as simulated by NCAR community climate model." Journal of Climate 4: (1991) 890-897.

Peters-Lidard, C. D., P. R. Houser, Y. Tian, S. V. Kumar, J. Geiger, S. Olden, L. Lighty, J. L. Eastman, B. Doty, P. Dirmeyer, J. Adams, K. Mitchell, E.F. Wood, and J. Sheffield. "High-performance Earth System modeling with NASA/GSFC's Land Information System." Innovations in Systems and Software Engineering 3, 3: (2007) 157-165.

Reichle, R. H., D. M. McLaughlin, and D. A. Entekhabi. "Hydrologic data assimilation with the Ensemble Kalman Filter." Monthly Weather Review 130, 1: (2002a) $103-114$. 
Reichle, R.H., W.T. Crow, and C.L. Keppenne. "An adaptive ensemble Kalman filter for soil moisture data assimilation." Water Resources Research 44, W03423: (2008) doi:10.1029/2007WR006,357.

Reichle, R.H., and R.D. Koster. "Assessing the impact of horizontal error correlations in background fields on soil moisture estimation." Journal of Hydrometeorology 4, 6: (2003) 1229-1242.

—. "Bias reduction in short records of satellite soil moisture." Geophysical Research Letters 31, 19: (2004) L19,501.

—. "Global assimilation of satellite surface soil moisture retrievals into the NASA Catchment land surface model." Geophysical Research Letters 32, 2: (2005) doi:10.1029/2004GL021,700.

Reichle, R.H., R.D. Koster, P. Liu, S.P.P. Mahanama, E.G. Njoku, and M. Owe. "Comparison and assimilation of global soil moisture retrievals from the Advanced Microwave Scanning Radiometer for the Earth Observing system (AMSR-E) and the Scanning Multichannel Microwave Radiometer (SMMR)." Journal of Geophysical Research - Atmospheres 112, D09108: (2007) doi:10.1029/2006JD008,033.

Reichle, R.H., J.P. Walker, R.D. Koster, and P.R. Houser. "Extended vs. Ensemble Kalman Filtering for Land Data Assimilation." Journal of Hydrometeorology 3, 6: (2002b) 728-740.

Rodell, M., P. R. Houser, U. Jambor, J. Gottschalck, K. Mitchell, C.-J. Meng., K. Arsenault, B. Cosgrove, J. Radakovich, M. Bosilovich, J. K. Entin, J. P. Walker, 
D. Lohmann, and D. Toll. "The Global Land Data Assimilation System." Bulletin of the American Meteorological Society 85, 3: (2004) 381-394.

Schmugge, T. J., T. J. Jackson, and H. L. McKim. "Survey of methods for soil moisture determination." Water Resources Research 16: (1980) 961-979.

Space Studies Board, NRC. Earth science and applications from space: National imperatives for the next decade and beyond. National Academy of Sciences, 2007.

Trier, S.B., F. Chen, and K.W. Manning. "A Study of convection initiation in a mesoscale model using high resolution land surface initial conditions." Monthly Weather Review 132: (2004) 2954-2976.

Walker, J. P., G. R. Willgoose, and J. D. Kalma. "One-dimensional soil moisture profile retrieval by assimilation of near-surface observations: a simplified soil moisture model and field application." Journal of Hydrometeorology 2, 4: (2001) 356-373.

—. "Three-dimensional soil moisture profile retrieval by assimilation of nearsurface measurements: simplified kalman filter covariance forecasting and field application." Water Resources Research 38, 12: (2002) doi:10.1029/2002WR001,545.

Zhou, Y., D. McLaughlin, and D. Entekhabi. "Assessing the performance of the ensemble kalman filter for land surface data assimilation." Monthly Weather Review 134: (2006) 2128-2142. 
Table 1a

Perturbation parameters for downward shortwave radiation (SW), downward longwave radiation (LW), and precipitation $(\mathrm{P})$ forcings.

\begin{tabular}{|l|l|lll|}
\hline \multirow{1}{*}{} & & \multicolumn{3}{|c|}{ Cross Correlations } \\
Variable & \multirow{2}{*}{ Stddev } & SW & LW & P \\
\cline { 3 - 5 } SW & $0.30[-]$ & 1.0 & -0.5 & -0.8 \\
LW & $50 \mathrm{~W} \mathrm{~m}^{-2}$ & -0.5 & 1.0 & 0.5 \\
$\mathrm{P}$ & $0.50[-]$ & -0.8 & 0.5 & 1.0 \\
\hline
\end{tabular}

Table $1 b$

Perturbation parameters for Catchment model prognostic variables. Cross-correlations are not imposed.

\begin{tabular}{|l|l|}
\hline Variable & Stddev $[\mathrm{mm}]$ \\
\hline catchment deficit & 0.14 \\
surface excess & 0.03 \\
\hline
\end{tabular}


Table 1c

Perturbation parameters for Mosaic soil moisture (sm) prognostic variables. Variable $\mathrm{sm}_{1}$ represents top-most layer.

\begin{tabular}{|l|l|lll|}
\hline & & \multicolumn{3}{|c|}{ Cross Correlations } \\
\cline { 3 - 5 } & \multirow{2}{*}{ Stddev } & \multicolumn{2}{|c|}{ with Perturbations in } \\
\cline { 3 - 5 } & & $\left.\mathrm{sm}_{1} \mathrm{~m}^{-3}\right]$ & $\mathrm{sm}_{2}$ & $\mathrm{sm}_{3}$ \\
\hline $\mathrm{sm}_{1}$ & $1.70 \mathrm{E}-3$ & 1.0 & 0.6 & 0.3 \\
$\mathrm{sm}_{2}$ & $1.50 \mathrm{E}-4$ & 0.6 & 1.0 & 0.6 \\
$\mathrm{sm}_{3}$ & $1.00 \mathrm{E}-4$ & 0.3 & 0.6 & 1.0 \\
\hline
\end{tabular}

Table 1d

Same as Table 1c but for Noah

\begin{tabular}{|l|l|llll|}
\hline \multirow{2}{*}{} & & \multicolumn{5}{|c|}{ Cross Correlations } \\
\cline { 3 - 6 } Variable & \multirow{2}{*}{ Stddev } & \multicolumn{4}{|c|}{ with Perturbations in } \\
\cline { 3 - 6 } & {$\left[\mathrm{m}^{3} \mathrm{~m}^{-3}\right]$} & $\mathrm{sm}_{1}$ & $\mathrm{sm}_{2}$ & $\mathrm{sm}_{3}$ & $\mathrm{sm}_{4}$ \\
\hline $\mathrm{sm}_{1}$ & $6.00 \mathrm{E}-3$ & 1.0 & 0.6 & 0.4 & 0.2 \\
$\mathrm{sm}_{2}$ & $1.10 \mathrm{E}-4$ & 0.6 & 1.0 & 0.6 & 0.4 \\
$\mathrm{sm}_{3}$ & $6.00 \mathrm{E}-5$ & 0.4 & 0.6 & 1.0 & 0.6 \\
$\mathrm{sm}_{4}$ & $4.00 \mathrm{E}-5$ & 0.2 & 0.4 & 0.6 & 1.0 \\
\hline
\end{tabular}


Table 1e

Same as Table 1c but for CLM

\begin{tabular}{|c|c|c|c|c|c|c|c|c|c|c|c|}
\hline \multirow[b]{2}{*}{ Variable } & \multirow{2}{*}{$\begin{array}{l}\text { Stddev } \\
{\left[\mathrm{m}^{3} \mathrm{~m}^{-3}\right]}\end{array}$} & \multicolumn{10}{|c|}{$\begin{array}{l}\text { Cross Correlations } \\
\text { with Perturbations in }\end{array}$} \\
\hline & & $\mathrm{sm}_{1}$ & $\mathrm{sm}_{2}$ & $\mathrm{sm}_{3}$ & $\mathrm{sm}_{4}$ & $\mathrm{sm}_{5}$ & $\mathrm{sm}_{6}$ & $\mathrm{sm}_{7}$ & $\mathrm{sm}_{8}$ & $\mathrm{sm}_{9}$ & $\mathrm{sm}_{10}$ \\
\hline $\mathrm{sm}_{1}$ & $1.00 \mathrm{E}-3$ & 1.0 & 0.7 & 0.7 & 0.6 & 0.6 & 0.6 & 0.6 & 0.4 & 0.4 & 0.4 \\
\hline $\mathrm{sm}_{2}$ & $7.00 \mathrm{E}-4$ & 0.7 & 1.0 & 0.7 & 0.7 & 0.6 & 0.6 & 0.6 & 0.6 & 0.4 & 0.4 \\
\hline $\mathrm{sm}_{3}$ & $5.00 \mathrm{E}-4$ & 0.7 & 0.7 & 1.0 & 0.7 & 0.7 & 0.6 & 0.6 & 0.6 & 0.6 & 0.4 \\
\hline $\mathrm{sm}_{4}$ & $3.00 \mathrm{E}-4$ & 0.6 & 0.7 & 0.7 & 1.0 & 0.7 & 0.7 & 0.6 & 0.6 & 0.6 & 0.6 \\
\hline $\mathrm{sm}_{5}$ & $2.00 \mathrm{E}-5$ & 0.6 & 0.6 & 0.7 & 0.7 & 1.0 & 0.7 & 0.7 & 0.6 & 0.6 & 0.6 \\
\hline $\mathrm{sm}_{6}$ & $2.00 \mathrm{E}-5$ & 0.6 & 0.6 & 0.6 & 0.7 & 0.7 & 1.0 & 0.7 & 0.7 & 0.6 & 0.6 \\
\hline $\mathrm{sm}_{7}$ & $2.00 \mathrm{E}-5$ & 0.6 & 0.6 & 0.6 & 0.6 & 0.7 & 0.7 & 1.0 & 0.7 & 0.7 & 0.6 \\
\hline $\mathrm{sm}_{8}$ & $1.50 \mathrm{E}-6$ & 0.4 & 0.6 & 0.6 & 0.6 & 0.6 & 0.7 & 0.7 & 1.0 & 0.7 & 0.7 \\
\hline $\mathrm{sm}_{9}$ & $1.50 \mathrm{E}-6$ & 0.4 & 0.4 & 0.6 & 0.6 & 0.6 & 0.6 & 0.7 & 0.7 & 1.0 & 0.7 \\
\hline $\mathrm{sm}_{10}$ & $5.00 \mathrm{E}-8$ & 0.4 & 0.4 & 0.4 & 0.6 & 0.6 & 0.6 & 0.6 & 0.7 & 0.7 & 1.0 \\
\hline
\end{tabular}




\section{Table 2}

Normalized information contribution (NIC) values of assimilated surface soil moisture to skill in surface soil moisture anomalies. Columns indicate which LSM is used in the generation of the synthetic truth and retrievals, and rows indicate which model is used to assimilate the synthetic retrievals. Last row and column indicate averages across all models.

\begin{tabular}{l|l|l|l|l|l}
\hline \hline \multirow{2}{*}{ Surface } & \multicolumn{4}{|c}{ TRUTH } \\
\hline & Catchment & Mosaic & Noah & CLM & Avg \\
\hline & 0.71 & 0.44 & 0.39 & 0.33 & 0.47 \\
Catchment & 0.43 & 0.59 & 0.54 & 0.57 & 0.53 \\
Mosaic & 0.40 & 0.44 & 0.53 & 0.45 & 0.46 \\
Noah & 0.37 & 0.52 & 0.45 & 0.67 & 0.50 \\
CLM & 0.48 & 0.50 & 0.48 & 0.49 & \\
\hline Avg & & & & & \\
\hline \hline
\end{tabular}


Table 3

Same as Table 3 but for root zone soil moisture NIC values.

\begin{tabular}{l|l|l|l|l|l}
\hline \hline \multirow{2}{*}{ Root zone } & \multicolumn{5}{|c}{ TRUTH } \\
\hline & Catchment & Mosaic & Noah & CLM & Average \\
\hline Catchment & 0.72 & 0.54 & 0.37 & 0.38 & 0.50 \\
Mosaic & 0.55 & 0.70 & 0.32 & 0.34 & 0.48 \\
Noah & 0.44 & 0.36 & 0.44 & 0.26 & 0.38 \\
CLM & 0.11 & 0.22 & 0.11 & 0.45 & 0.22 \\
\hline Average & 0.46 & 0.48 & 0.29 & 0.36 & \\
\hline \hline
\end{tabular}



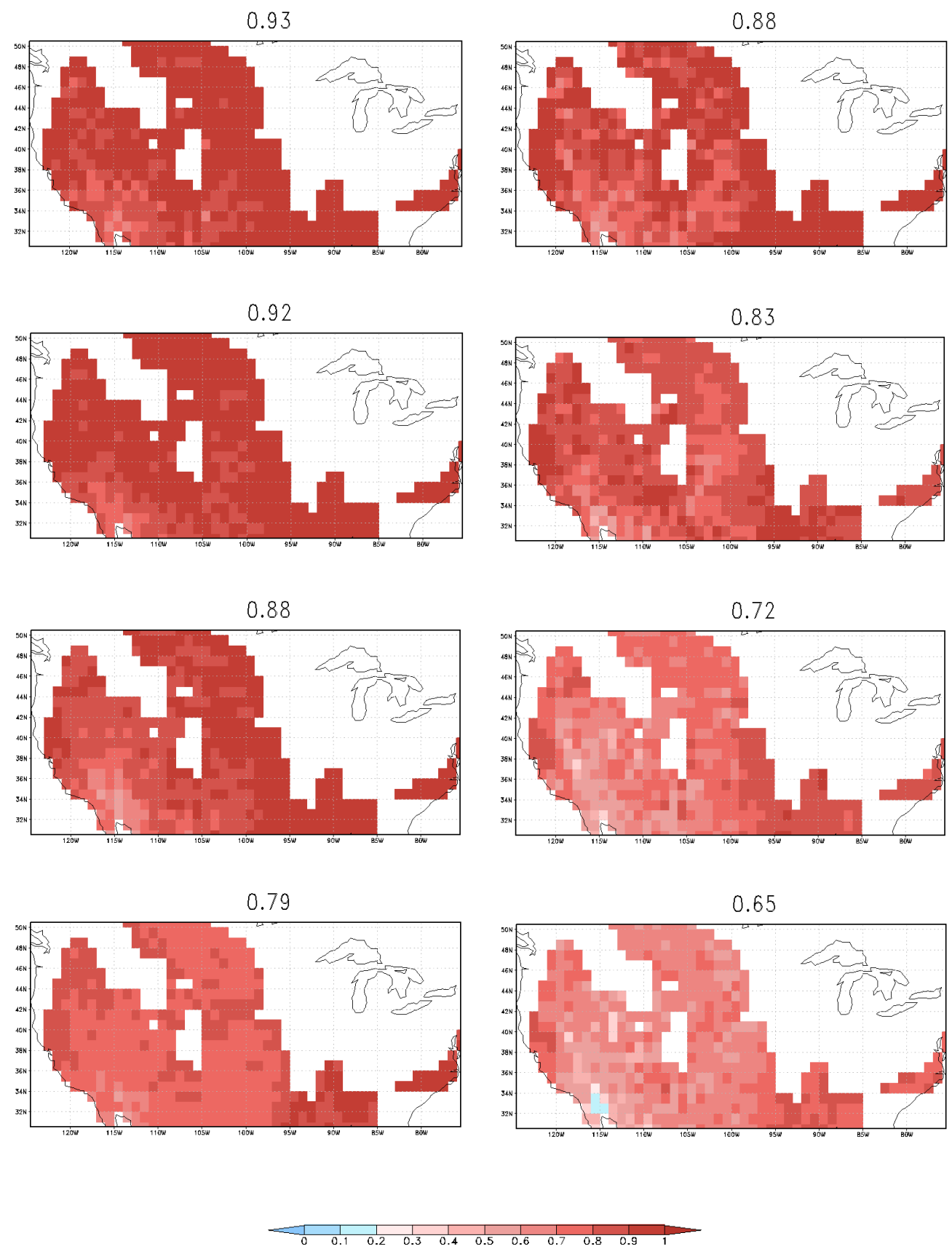

Fig. 1. (Left column) Anomaly time series correlation coefficient ("native vertical coupling strength") between surface and root zone soil moisture and (right column) time average gain correlations from the assimilation experiments for the four LSMs (the rows from top to bottom represent Catchment, Mosaic, Noah and CLM, respectively). Titles show domain averaged values. 

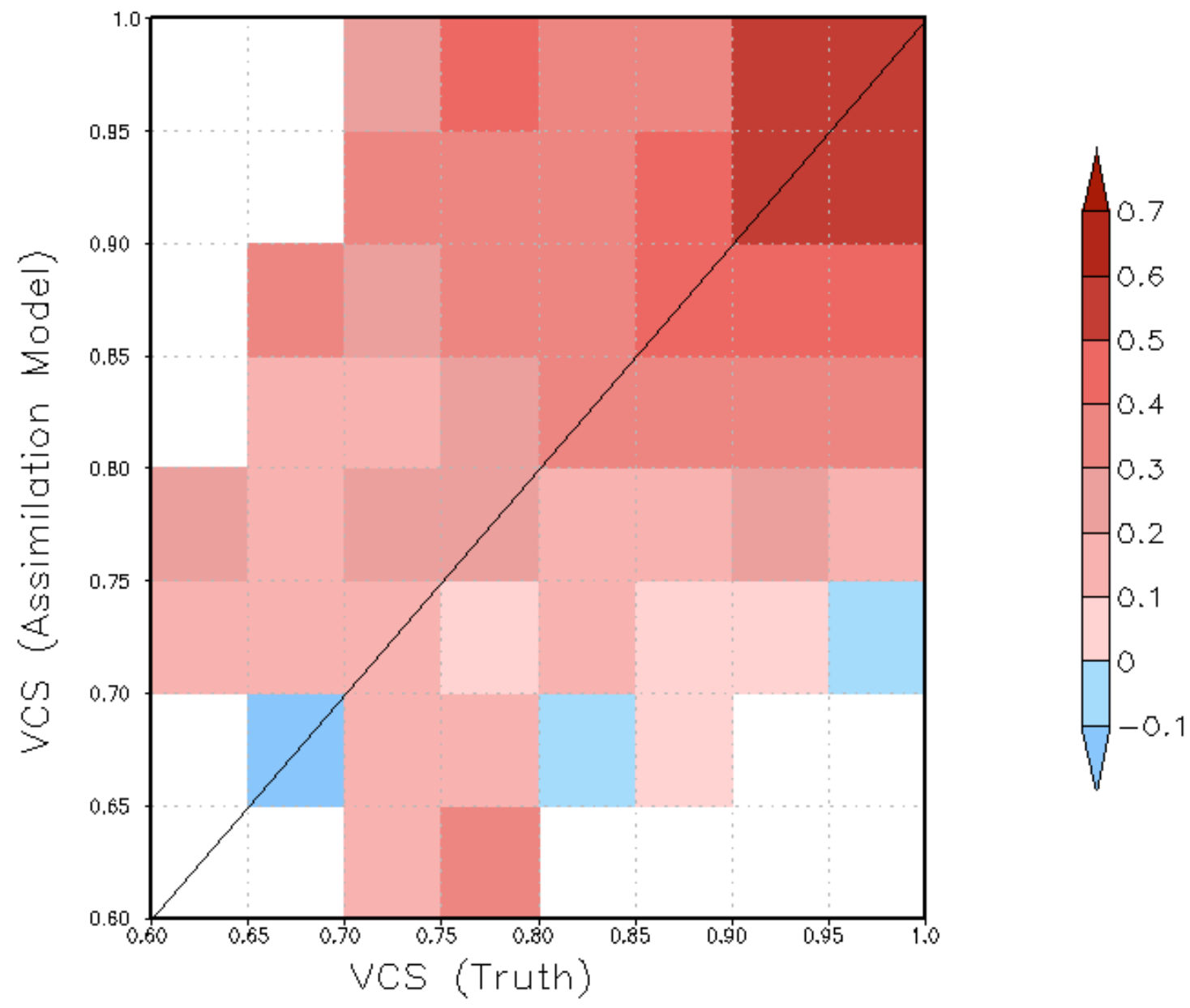

Fig. 2. Normalized information contribution from the fraternal twin assimilation runs as function of the (abscissa) native vertical coupling strength of the model used for generating truth and (ordinate) native vertical coupling strength of the model used in the assimilation integration. 


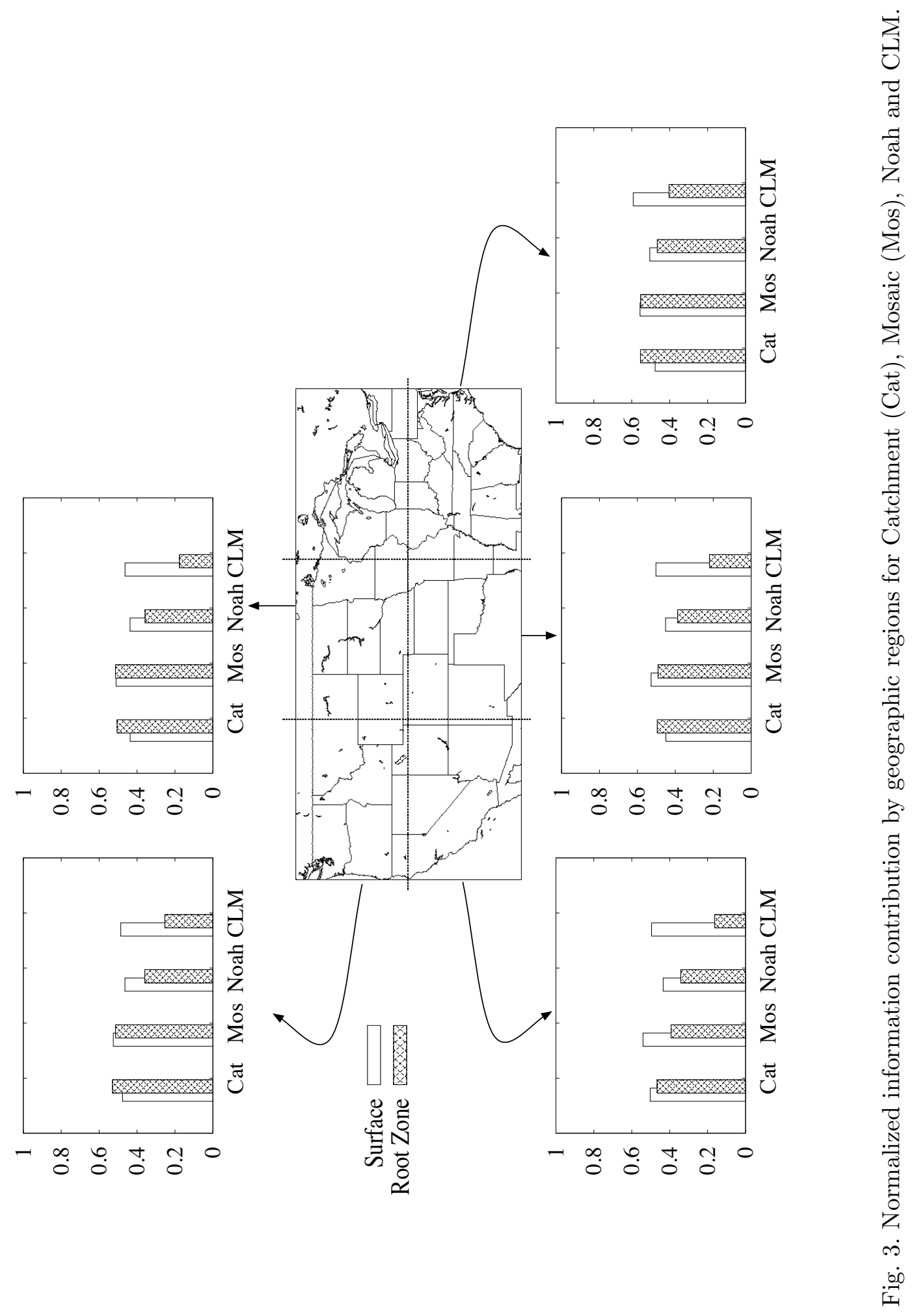



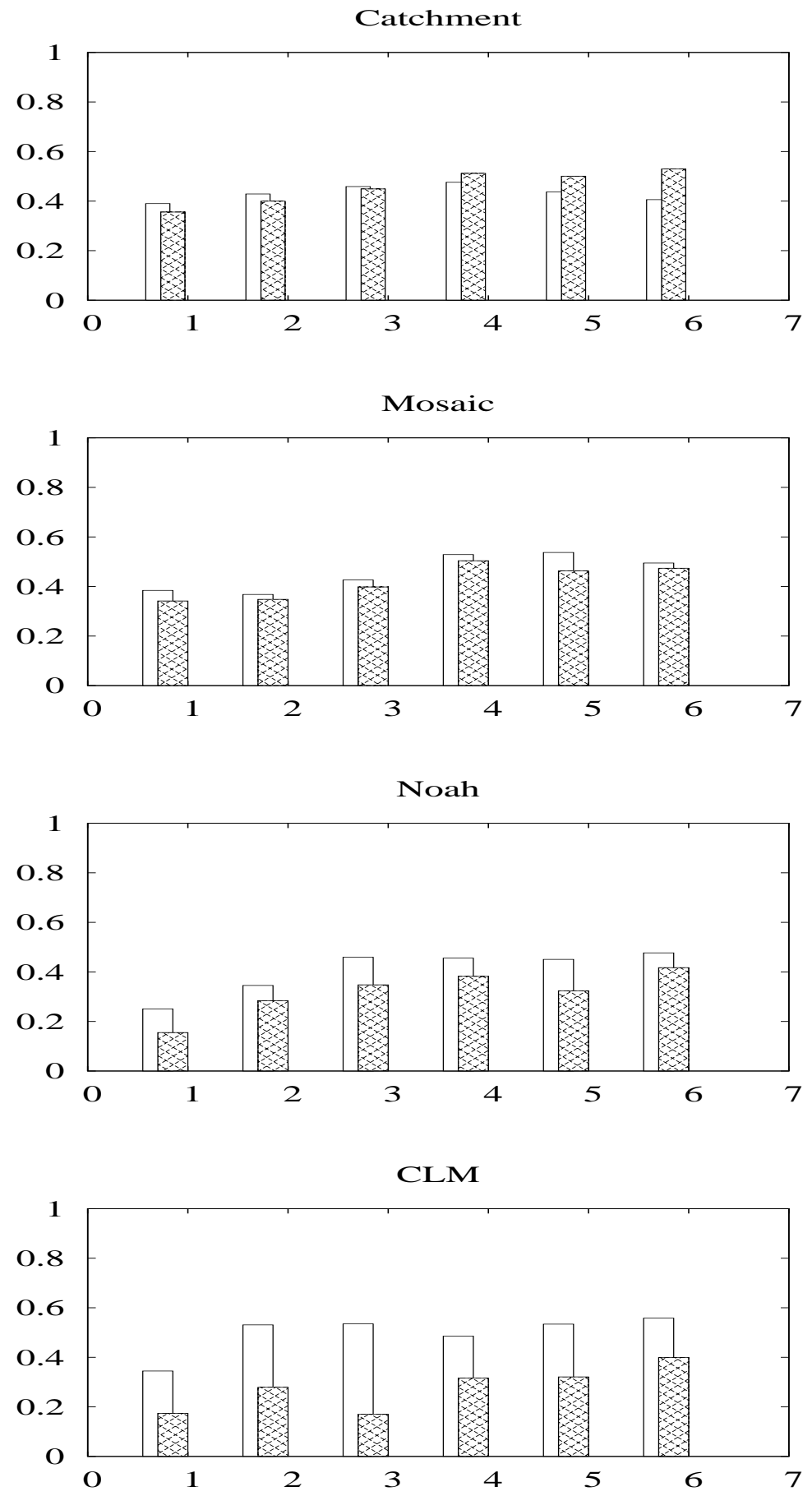

Fig. 4. Normalized information contribution by soil texture (1 - Loamy sand, 2 - Sandy loam, 3- Sandy clay loam, 4 - Loam, 5 - Clay loam, 6 - Clay) for Catchment, Mosaic, Noah, and CLM. 